\title{
Novel G9a/DNMT first-in-class dual reversible inhibitor has potent antitumor effect in bladder cancer
}

\author{
Whi-An Kwon ${ }^{1}$, Ho Kyung Seo ${ }^{2}$ \\ ${ }^{1}$ Department of Urology, Myongji Hospital, Hanyang University College of Medicine, Goyang, Gyeonggi-do, Korea; ${ }^{2}$ Department of Urology, \\ Center for Urologic Cancer, Hospital/Division of Tumor Immunology, Research Institute, National Cancer Center, Goyang, Gyeonggi-do, Korea \\ Correspondence to: Ho Kyung Seo, MD, PhD. Department of Urology, Center for Urologic Cancer, Hospital/Division of Tumor Immunology, \\ Research Institute, National Cancer Center, 323 Ilsan-ro, Ilsandong-gu, Goyang-si, Gyeonggi-do, 410-769, Korea. Email: seohk@ncc.re.kr. \\ Comment on: Segovia C, San José-Enériz E, Munera-Maravilla E, et al. Inhibition of a G9a/DNMT network triggers immune-mediated bladder \\ cancer regression. Nat Med 2019;25:1073-81.
}

Submitted Oct 09, 2019. Accepted for publication Jan 06, 2020.

doi: $10.21037 /$ tcr.2020.01.16

View this article at: http://dx.doi.org/10.21037/tcr.2020.01.16

Epigenetic changes, which are heritable changes that alter gene expression without changing the primary DNA sequence, are a hallmark of cancer. The epigenome is a therapeutic target in cancer. There are two main forms of epigenetic modifications: those that directly modify DNA (i.e., DNA methylation) and those that modify DNAbinding proteins (i.e., histone modifications).

G9a is overexpressed in various cancers. The methylation of its target, histone 3 lysine 9 (H3K9), is correlated with silence of transcription (1-3). Some researchers have reported that suppression of G9a expression leads to decreased tumor cell proliferation, delayed cancer progression, and blocked tumor metastasis $(1,2,4,5)$. In addition, G9a interacts with DNA methyltransferase-1 (DNMT1) to regulate methylation of DNA and histone while cell division, which promotes transcriptional silence of the target gene $(6,7)$. A decrease in methylation levels of DNA and $\mathrm{H} 3 \mathrm{~K} 9$ promote reactivation of tumor suppressor genes that suppress tumor cell growth $(8,9)$. However, the basic mechanism is not fully understood.

CM-272 is a first-in-class potent, dual and reversible inhibitor of G9a (GLP) and DNMT with novelty. The article of Segovia et al. (10) presents a potential new strategy for the treatment of bladder cancer (BC) from the use of an epigenetic inhibitor which previously unknown in combination with immune checkpoint inhibitors (ICIs).

\section{Role of G9a/DNA methyltransferase-1 in bladder cancer}

G9a (EHMT2), a histone methyltransferase that catalyzes lysine 9 of histone 3 (H3 K9), showed high levels of expression in several malignancies like breast cancer and head and neck squamous cell carcinoma. Previous studies reported that G9a was highly expressed in bladder transitional cell carcinoma (TCC), and that G9a inhibition notably diminished cell proliferation (11). These results have attracted attention towards the role of G9a in cellular metabolism and suggest that G9a may be a therapeutic target for bladder TCC. Although G9a overexpression in non-muscle-invasive bladder cancer (NMIBC) is associated with poor clinical outcomes, recurrence in NMIBC is influenced by many confounding factors. Hence, further analysis is needed to correlate G9a RNA levels with NMIBC prognosis. Studies are also needed on G9a RNA levels to determine its association with molecular subtypes and with their prognosis.

Use of specific G9a (A-366) and DNMT (decitabine) inhibitors in combination, or use of small interfering RNA (siRNA)has a synergistic effect.

\section{Mode of action of CM-272}

CM-272 is a novel reversible dual inhibitor against G9a and DNMTs. It lengthens survival in in vivo models of hematologic cancers by causing immunogenic cell death (12). While CM-272 does not directly affect EZH2 methyltransferase activity, it leads to a decrease in $\mathrm{H} 3 \mathrm{~K} 27 \mathrm{me} 3$ levels exclusively as well as $\mathrm{H} 3 \mathrm{~K} 9 \mathrm{me} 2$ levels in BC. Knockdown or overexpression of EZH2 confers elevated resistance or sensitivity, respectively, to CM-272 in 
BC. Cumulatively, above results represent the presence of a regulatory loop between $\mathrm{EZH} 2$ and $\mathrm{G} 9 \mathrm{a}$ in $\mathrm{BC}$, without direct effect of CM-272 on methyltransferase activity of $\mathrm{EZH} 2$.

Segovia and colleagues report that CM-272 induced upregulation of genes involved in the regulation of immune responses in $\mathrm{BC}$ cell lines, and that the combination of CM-272 and anti-PD-L1 was effective against primary and metastatic tumors in mice, producing a sustained reduction in, or elimination of, tumor burden (10).

\section{Clinical implications of CM-272 in BC treatment}

For decades, standard treatment for advanced urothelial carcinoma (UC) has been focused almost exclusively on cisplatin-based chemotherapy. ICIs have been demonstrated to have a durable long-term response and tolerable safety in recent clinical trials. However, in about $70-80 \%$ of patients with metastatic BC the tumor is unresponsive to ICIs. Therefore, further research on the inhibition of G9a/DNMT network in BC could present a potential new strategy for the treatment of $\mathrm{BC}$ from the use of a previously unknown epigenetic inhibitor in combination with ICIs.

Segovia et al. (10) demonstrated that treatment of CM$272+$ cisplatin shows statistically significant regression in immunocompetent quadruple-knockout (PtenloxP/loxP; Trp531oxP/loxP; Rb1loxP/loxP; $\mathrm{Rbl1}^{-/}$) transgenic mouse models. However, the immune system of the mice in this study is different from that of humans. Therefore, this effect needs to be demonstrated again in the humanized mouse model.

The treatment of CM-272 + cisplatin reveals statistically significant regression of established cancers and metastases. Recent studies $(12,13)$ have shown that the responses of type I interferon impact to the efficacy of chemotherapeutic agents. This could be one of the mechanisms of action of CM-272 + cisplatin treatment.

The anticancer effect is notably enhanced with the combination of CM-272 with anti-programmed cell death ligand 1 (PD-L1), even in the absence of cisplatin. These effects correlate with an endogenous anticancer immune response and immunogenic cell death, with the conversion of a cold tumor into a hot tumor. The PD-L1 expression change after CM-272 treatment in vivo needs to be studied further.

In the study of Segovia et al. (10), on day $28,75 \%$ of the mice in the anti-PD-L1 treatment group had evidence of cancers and metastases, whereas only $17 \%$ of the mice in the CM-272 + anti-PD-L1 treatment group, with or without cisplatin (CDDP)-based chemotherapy, had evidence of a primary cancer or metastatic disease. To enhance the effects of ICIs, extensive studies are being conducted to investigate the therapeutic effects of combinations of anticancer drugs and ICIs. Therefore, Segovia et al. (10) need to explain whether the effect of treatment with CM-272 plus antiPD-L1 plus CDDP is lower than that of CM-272 + antiPD-L1 due to renal toxicity or other reasons.

The recent National Comprehensive Cancer Network (NCCN) Guideline recommends the use of ICIs as the primary treatment in CDDP-ineligible patients whose tumors express high levels of PD-L1 (14).

Carboplatin-based combination chemotherapy is recommended when PD-L1 expression is not increased, in which case an objective response rate of about $30-40 \%$ for 9 to 10 months has been reported (15). This study included a cohort of CDDP-ineligible patients (1 responder and 2 non-responders) with advanced or metastatic UC who were treated with first-line anti-PD-1 therapy. In order to interpret the results more accurately, additional information about PD-L1 expression may be required.

G9a expression was associated with resistance to programmed cell death protein 1 (PD-1) inhibition in patients with BC. Further research is needed to determine whether G9a expression can be used to predict the effects of ICIs.

\section{Conclusions}

Targeting or modulating G9a/DNMT methyltransferase activity might emerge as a new area in the treatment of BC. The results of the study of Segovia et al. (10) have furthered the implications of investigating G9a/DNMT. Validation of CM-272-induced up-regulation of genes involved in the regulation of immune responses in a larger cohort of patients with BC could lead to the discovery of a new combination therapy of CM-272 and ICIs and provide biomarkers for immunotherapy in patients with metastatic BC.

\section{Acknowledgments}

We would like to thank Editage (www.editage.co.kr) for English language editing.

Funding: This study was supported by a Korean National 
Cancer Center grant (NCC1810242).

\section{Footnote}

Provenance and Peer Review: This article was commissioned and reviewed by the Section Editor Peng Zhang (Department of Urology, Zhongnan Hospital of Wuhan University, Wuhan, China).

Conflicts of Interest: Both authors have completed the ICMJE uniform disclosure form (available at http://dx.doi. org/10.21037/tcr.2020.01.16). The authors have no conflicts of interest to declare.

Ethical Statement: The authors are accountable for all aspects of the work in ensuring that questions related to the accuracy or integrity of any part of the work are appropriately investigated and resolved.

Open Access Statement: This is an Open Access article distributed in accordance with the Creative Commons Attribution-NonCommercial-NoDerivs 4.0 International License (CC BY-NC-ND 4.0), which permits the noncommercial replication and distribution of the article with the strict proviso that no changes or edits are made and the original work is properly cited (including links to both the formal publication through the relevant DOI and the license). See: https://creativecommons.org/licenses/by-nc-nd/4.0/.

\section{References}

1. Hua KT, Wang MY, Chen MW, et al. The H3K9 methyltransferase G9a is a marker of aggressive ovarian cancer that promotes peritoneal metastasis. Mol Cancer 2014;13:189.

2. Chen MW, Hua KT, Kao HJ, et al. H3K9 histone methyltransferase G9a promotes lung cancer invasion and metastasis by silencing the cell adhesion molecule EpCAM. Cancer Res 2010;70:7830-40.

3. Zhong X, Chen X, Guan X, et al. Overexpression of G9a and MCM7 in oesophageal squamous cell carcinoma is associated with poor prognosis. Histopathology 2015;66:192-200.

4. Zhang J, He P, Xi Y, et al. Down-regulation of G9a triggers DNA damage response and inhibits colorectal cancer cells proliferation. Oncotarget 2015;6:2917-27.

5. Lehnertz B, Pabst C, Su L, et al. The methyltransferase
G9a regulates HoxA9-dependent transcription in AML. Genes Dev 2014;28:317-27.

6. Esteve PO, Chin HG, Smallwood A, et al. Direct interaction between DNMT1 and G9a coordinates DNA and histone methylation during replication. Genes Dev 2006;20:3089-103.

7. Tachibana M, Matsumura Y, Fukuda M, et al. G9a/ GLP complexes independently mediate $\mathrm{H} 3 \mathrm{~K} 9$ and DNA methylation to silence transcription. EMBO J 2008;27:2681-90.

8. Wozniak RJ, Klimecki WT, Lau SS, et al. 5-Aza-2'deoxycytidine-mediated reductions in G9A histone methyltransferase and histone $\mathrm{H} 3 \mathrm{~K} 9$ di-methylation levels are linked to tumor suppressor gene reactivation. Oncogene 2007;26:77-90.

9. Sharma S, Gerke DS, Han HF, et al. Lysine methyltransferase $\mathrm{G} 9 \mathrm{a}$ is not required for DNMT3A/3B anchoring to methylated nucleosomes and maintenance of DNA methylation in somatic cells. Epigenetics Chromatin 2012;5:3.

10. Segovia C, San Jose-Eneriz E, Munera-Maravilla E, et al. Inhibition of a G9a/DNMT network triggers immune-mediated bladder cancer regression. Nat Med 2019;25:1073-81.

11. Li F, Zeng J, Gao Y, et al. G9a Inhibition Induces Autophagic Cell Death via AMPK/mTOR Pathway in Bladder Transitional Cell Carcinoma. PLoS One 2015;10:e138390.

12. San Jose-Eneriz E, Agirre X, Rabal O, et al. Discovery of first-in-class reversible dual small molecule inhibitors against G9a and DNMTs in hematological malignancies. Nat Commun 2017;8:15424.

13. Sistigu A, Yamazaki T, Vacchelli E, et al. Cancer cellautonomous contribution of type I interferon signaling to the efficacy of chemotherapy. Nat Med 2014;20:1301-9.

14. National Comprehensive Cancer Network. Bladder cancer (Version 4.2019). Available online: https://www.nccn.org/ professionals/physician_gls/pdf/bladder.pdf. Accessed September 30, 2019.

15. Kim HS, Seo HK. Immune checkpoint inhibitors for urothelial carcinoma. Investig Clin Urol 2018;59:285-96.

Cite this article as: Kwon WA, Seo HK. Novel G9a/DNMT first-in-class dual reversible inhibitor has potent antitumor effect in bladder cancer. Transl Cancer Res 2020;9(3):13191321. doi: 10.21037/tcr.2020.01.16 